\title{
Pinna Movements of the Cat during Sound Localization
}

\author{
Luis C. Populin and Tom C. T. Yin \\ Neuroscience Training Program and Department of Neurophysiology, University of Wisconsin-Madison, Madison, \\ Wisconsin 53706
}

\begin{abstract}
We measured the movements of the external ear, or pinna, using the magnetic search coil technique in cats trained to look at auditory and visual targets for a food reward. No behavioral contingencies were placed on pinna movements. Prominent pinna movements accompany eye movements when the animal orients to either auditory or visual stimuli. In visual trials the pinna movements are coordinated with eye movements, suggesting that they are part of the general orientation response of the animal. In auditory trials the pinna response was composed
\end{abstract}

of two movements: short- and long-latency components. Whereas the long-latency component seemed to occur with the eye movement to the target, the short-latency component was coupled to the onset of the stimulus. The short-latency component ( $\sim 25 \mathrm{msec}$ ) was highly asymmetrical, being largest in the pinna ipsilateral to the stimuli. In one animal it persisted after $>10^{5}$ trials.

Key words: cat; external ear; pinna movements; sound localization; orientation response; auditory and visual stimuli
Acoustical evidence and psychophysical evidence indicate that the pinna plays an important role in sound localization, particularly for sources on the midsagittal plane that generate minimal interaural disparities. The passive acoustical properties of this structure, along with the head and torso, attenuate or amplify specific frequencies in a directional manner (Wiener et al., 1966; Phillips et al., 1982; Calford and Pettigrew, 1984; Musicant et al., 1990; Rice et al., 1992), which for broadband signals results in unique spectral profiles that are known as head-related transfer functions (HRTFs). Which spectral features (notches, peaks, or some combination) imposed by the pinna are used to help determine the location of sound remain to be determined (Middlebrooks, 1992).

In humans, a species with relatively immobile ears, the HRTF is coupled to head position, resulting in a one-to-one correspondence between the spectral profile of broadband sources and the subject's head (Wightman and Kistler, 1989). In species with mobile ears such as the cat, the pinnae can be oriented independently of head position, breaking the one-to-one correspondence between acoustic sources and the head. The question arises, therefore, regarding what role (if any) the mobility of the pinna plays in sound localization in these species.

On the one hand the mobility of the pinna may complicate the localization process by increasing the computational load, because the position of the structure must be taken into account. On the other hand, it can aid localization by allowing the animal (1) to obtain multiple samples of an acoustic object (Thurlow and Runge, 1967) and (2) to separate the spectrum of a sound source from the HRTF (Young et al., 1996).

Despite the potential implications of pinna movements for sound localization (Phillips and Brugge, 1985), the functional role

Received Jan. 21, 1998; revised March 10, 1998; accepted March 12, 1998.

This work was supported by National Institutes of Health Grants DC00116 and DC02840. We thank Ravi Kochhar and Jane Sekulski for computer programming, Richard Olson for electronics, and Donna Cole for animal care and help with the experiments.

Correspondence should be addressed to Luis C. Populin, Department of Anatomy, University of Wisconsin, 1300 University Avenue, Madison, WI 53706.

Copyright (ㄷ) 1998 Society for Neuroscience $0270-6474 / 98 / 184233-11 \$ 05.00 / 0$ of pinna movements has received little attention, judging by the extent of published experimental work. There is direct evidence of an effect of pinna position on the receptive fields of superior colliculus (SC) auditory neurons (Middlebrooks and Knudsen, 1987), but the available behavioral evidence is indirect and contradictory. Heffner and Heffner (1988) assumed that longduration stimuli would allow time for the animal to move its pinnae, but they found no difference in localization acuity between short- and long-duration stimuli in the cat. On the other hand, Jenkins and Masterton (1982) found that monaural localization improved with long-duration stimuli. In none of the previous studies was the amplitude or timing of pinna movements measured despite their potential for modifying the acoustic stimuli.

In the present work we recorded pinna movements in cats while they performed various sound localization tasks (Populin and Yin, 1998). Because of difficulties in measuring absolute pinna position, our analyses focused on the temporal aspects of these movements.

Preliminary results have been presented in abstract form (Populin and Yin, 1997a).

\section{MATERIALS AND METHODS}

A detailed description of the training paradigm and experimental procedures is found in a preceding paper (Populin and Yin, 1998). Briefly, using the magnetic search coil technique (Fuchs and Robinson, 1966) to measure eye movements, we trained cats to look at the location of sound sources with their heads fixed. Auditory and visual stimuli were presented from an array of speaker and light-emitting diode (LED) assemblies located in front of the cat. Acoustic stimuli were broadband (0.1-25 $\mathrm{kHz}$ ) noise bursts. The animals were food-deprived and rewarded when their eyes were within electronic windows surrounding the targets.

A typical session included a random mixture of various experimental tasks: visual and auditory fixations, standard and delayed saccades, and sensory probes. Not all tasks were used in every experimental session, but a variety was always presented to prevent the cat from anticipating upcoming trials.

Pinna movements recorded during standard and delayed saccades to visual and auditory targets (Populin and Yin, 1998) and auditory sensory probe trials are included in this report. In the standard saccade task the cat was required to first fixate an LED at the primary position $\left(0^{\circ}, 0^{\circ}\right)$ and then saccade to a spatially disparate visual or auditory target presented 

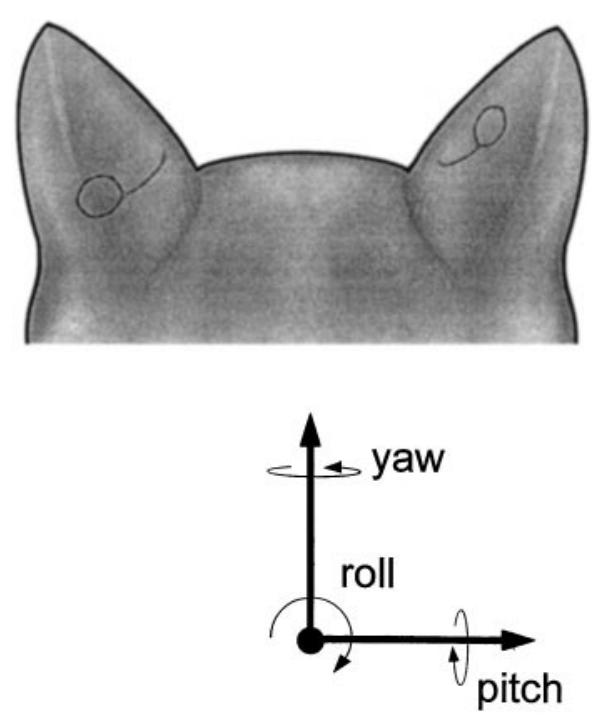

Figure 1. Posterior view of a cat's head illustrating the two coil positions used in these experiments. On the right the coil is shown in the flat medial aspect of the pinna, whereas on the left the coil is shown as far caudal on the pinna as possible. The vectors illustrate the convention used to describe changes in pinna position from a viewpoint directly behind the cat pointed in the same direction as the nose.

at the time the fixation LED was turned off. In the delayed saccade task the target was presented some time (500-700 msec) before the offset of the fixation LED, which constituted the signal for the cat to saccade. In sensory probe trials the fixation LED remained on for the entire duration, and the cat was expected to fixate it, even when an acoustic probe was presented during the fixation period. In saccade trials the cat was rewarded for making a saccade to the target within specified spatial and temporal windows (Populin and Yin, 1998), whereas in sensory probe trials the cat was rewarded for not breaking fixation. No behavioral contingencies were placed on pinna movements in any task.

Coil implant. Pinna movements were routinely recorded with coils anchored to the pinna. We considered two options for attaching the coil to the ear: taping the coil to the external aspect of the pinna before each session (Jay and Sparks, 1987; Hartline et al., 1995) and implanting it under the skin. We chose the latter because it permitted more stable recordings than a removable coil, which was subject to slippage during an experiment; it maximized reproducibility, because it would be impossible to attach the coil in the same position day after day; it minimized noise from loose leads; and it minimized discomfort for the cat, which is very particular about its pinnae. The disadvantages of implanting the coil were the potential for damaging the musculature, innervation, or blood supply and restricting pinna movements with taut coil leads. None of the cats implanted with this procedure showed any signs of discomfort or impaired pinna function. Furthermore, postmortem inspection indicated that the coil was firmly held in place. We conclude that the disadvantages were kept to a minimum.

The search coils were implanted subcutaneously under sterile surgical conditions at the same time that the head restraint was attached. In Cat06 we implanted the coil on the flat, medial aspect of the right pinna, because it would help preserve the geometry of the coil (Fig. 1, right ear) (see Figs. 7, 10). However, the resulting position of the coil was far from perpendicular to the magnetic fields and complicated the calibration procedure. The phase detectors of our magnetic search coil system (CNC Engineering) produce an output signal that is a sinusoidal function of angle; thus the output is highly nonlinear when the coil is oriented $\geq 30^{\circ}$ from the coronal plane. In subsequent cats we implanted the coils more caudally in the pinna, as close to the coronal plane as possible within the limitations imposed by its geometry (Fig. 1, left ear; see Figs. 4-6, 8, 9).

A 6-7 mm incision was made in the skin, and a pocket large enough to house the coil was dissected. Each coil was $10 \mathrm{~mm}$ in diameter and made of three turns of AS633 fine wire (Cooner Wire Co.). Precautions were taken to avoid damaging the delicate muscles, their innervation, or blood supply. The leads from the coils were routed loosely, so as not to tether the pinna, underneath the skin to the top of the head where they were soldered to brass connectors (Microtech Inc., Boothwyn, PA) embedded in dental cement near the head post.

To calibrate the pinna coils we made use of the observation that the cats would generally orient their pinnae to a more or less standard position when they fixated a visual stimulus at the primary position $\left(0^{\circ}, 0^{\circ}\right)$. The calibrations were then made around this position. The orientation of each pinna coil when the cat fixated at $\left(0^{\circ}, 0^{\circ}\right)$ was estimated by eye during behavioral testing with a calibrating ring (Fig. 2, cr). This ring, made of malleable copper wire, was carefully manipulated to overlie the implanted coil, the contour of which could be seen while the cat was fixating an LED at the primary position. Once the orientation of the ring was judged to match that of the pinna coil, the cat was removed from the experimental setup, and the angular deviation of the ring from the coronal plane was measured. A dummy coil, physically matched to those implanted, was placed at the same orientation in the center of the field coils and rotated along the $x$ - and $y$-axes separately, over a range of $\pm 20^{\circ}$. The voltage output of the coil system was sampled at $10^{\circ}$ intervals, and the data points relating angular deviation and voltage output were fit with linear equations. The coefficients were used by the data analysis programs to transform the voltage output of the actual pinna coil into angular deviation.

Data analysis. The onset of pinna movement was determined with a criterion similar to the one used previously for eye movements (Populin and Yin, 1998). Pinna movement onset was defined as the time at which the velocity trace was $>2$ SD of the mean velocity baseline, from 125 msec before to $5 \mathrm{msec}$ after the onset of the stimulus. The horizontal and vertical components were computed separately, and the component with the shorter latency was chosen for analyses. Because of the small amplitude of some pinna movements and large first derivatives of the noise in the traces of some trials, we had to override the objective criterion and pick the onset of movement by eye in $\sim 15-20 \%$ of the trials. A 2 msec acoustic delay was subtracted from all latency measurements. Statistical analyses are presented as means and confidence intervals.

\section{RESULTS}

Pinna movement data from six different cats were collected while they engaged in sound localization tasks in which they were free to move their ears (Populin and Yin, 1998). In all figures the $0^{\circ}$ on the ordinate scale was arbitrarily set to approximate the position of the pinna at the time the cat fixated the LED at the primary position for that recording session. Thus, the scales measure changes in pinna position rather than absolute position.

\section{Pinna movements to visual targets from two- dimensional video images}

We begin with videotaped images of a cat's pinnae to provide a qualitative reference for the pinna movements measured by the search coil method. Shown in Figure 2 are posterior views of Cat08's head illustrating the position of the pinnae during various phases of a downward visual saccade. These images were selected from a video recording of an experimental session. The camera was positioned behind the cat, slightly to the right of the sagittal plane, and above the interaural axis. The gray structure labeled $h h$ in Figure 2 is the head holder, and the circular structure labeled $\mathrm{cr}$ is a calibrating ring, both of which are stationary in these images (see Materials and Methods).

To better track pinna position, triangular markers were attached to the tip and medial and lateral edges of each pinna, and a white reference dot was painted on the skin at the center of the implanted coil. Changes in pinna position in these figures (Fig. 1, bottom) are described as changes in yaw (left or right movements around the vertical axis), pitch (up or down movements around the horizontal axis), and roll (movements around the longitudinal axis of the cat).

During a typical behavioral run there was a fixed intertrial interval (5-10 sec) during which the cat was usually licking the reward from the previous trial. When the cat anticipated that the next trial was about to begin, it usually looked near the primary 

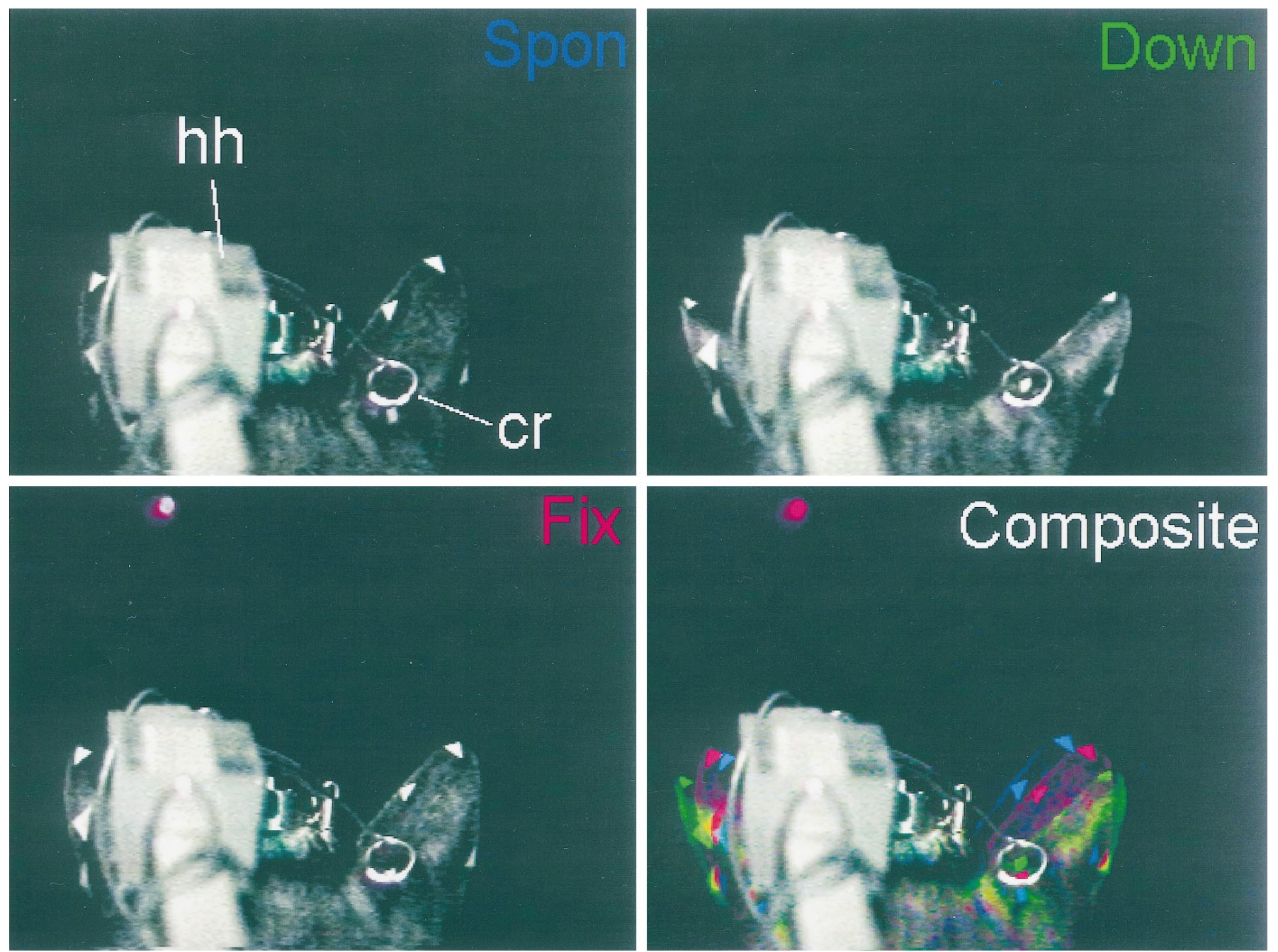

Figure 2. Images of a pinna movement to a visual target. Posterior views of Cat08's head from video recordings taken during an experimental session. Spon, Fix, and Down images were taken at the following points: during the spontaneous period before the onset of the trial (Spon), during the fixation period while fixating an LED at the primary position (Fix), and after the cat saccaded to the target (Down). The gray-white structure labeled $h h$ is the head holder, and the structure labeled $c r$ is the calibrating ring. Triangular markers were placed on the tip, medial, and lateral edges of each pinna, and the white dot on the right pinna marks the center of the implanted coil. Composite shows all three images superimposed in different colors (Spon, blue; Fix, red; Down, green).

position and pulled its pinnae into a ready position. The Spon image in Figure 2 was taken during this period, which we reserve for spontaneous activity in physiological recordings. The second image (Fig. 2, Fix) was taken while the cat was fixating the LED at the primary position, seen in the figure as a red dot. The third image (Fig. 2, Down) was taken after the cat had made a downward saccade to the LED at $\left(0^{\circ},-23^{\circ}\right)$. These images were selected from frames corresponding to each part of the task after the pinna had settled to a new position.

To show the relative movements of the pinna during these three sequences, we superimposed the three images in Figure 2, Composite. This figure was generated by decomposing each of the three images into its red, green, and blue components using Corel Photo Paint, keeping only one of the color components of each image (blue for Spon, red for Fix, and green for Down) and recombining them into a single image. In this way, the parts of the figure that remain stationary in all three images appear in their original color, and those that move appear in the color of the retained image or some combination of colors. The relative changes in pinna position can thus be seen in a single composite image.

Using the blue portion of the image from the spontaneous period as a reference, we will track the changes in pinna position throughout the sequence (Fig. 2). Notice that the dot marking the center of the coil is below the calibrating ring in the Spon period and is seen as a faint blue dot in the Composite image. In the Fix period the right pinna rolled laterally, as can be seen by the triangular markers on the pinna, and pitched slightly downward, as indicated by the shift in the position of the reference dot, which at this point is inside the lower edge of the calibrating ring. The left pinna also rolled laterally, although the details of its movement are obscured by the head holder.

In the last part of the task an LED was presented below the horizontal plane at $\left(0^{\circ},-23^{\circ}\right)$. The right pinna rolled further to the right and pitched downward, as indicated by the reference dot at the center of the calibrating ring and the almost complete disappearance of the medial triangular marker. The change in position of the left pinna appears to mirror that of the right pinna. 

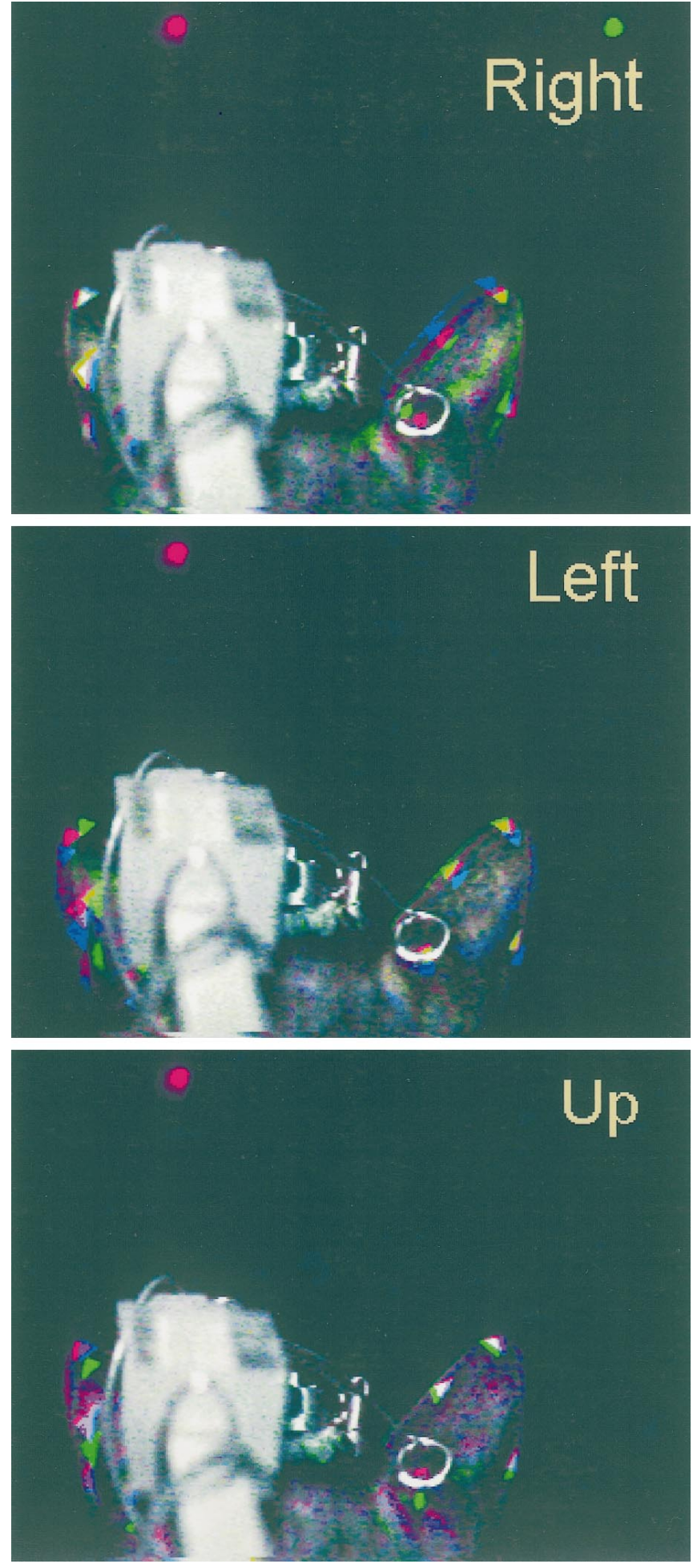

Figure 3. Composite figures illustrating the changes in pinna position when the cat saccaded to targets to the Right, Left, and Up. Details as in Figure 2.

Figure 3 shows composite images analogous to the one in Figure 2 for the other three targets with the same color convention. In Figure 3, top (for a target to the right at $18^{\circ}, 0^{\circ}$ ) the fixation LED is shown in red, whereas the target LED in green is also visible to the right. As in Figure 2, the right pinna rolled laterally between the spontaneous (blue) and fixation (red) periods. The target LED evoked an outward (yaw) movement to the right, as indicated by the green triangular marker in the medial aspect of the right pinna that has almost disappeared from view, the lateral marker that is more visible, and the medial movement of the reference dot (red to green) inside the calibrating ring. On the other hand, there was little change in the position of the left pinna throughout the entire trial, because the triangular markers are mostly white.

The initial part of the sequence for the Left target $\left(-18^{\circ}, 0^{\circ}\right)$ (Fig. 3) started with both pinnae rolled more laterally than in the previous case (blue image). The red image, taken during the fixation period, shows that the pinnae rolled slightly medially. The magnitude of the displacement is similar in both pinnae. The visual target presented $18^{\circ}$ to the left evoked different movements on the two sides; the contralateral right pinna rolled slightly to the left between the fixation and saccade period, as suggested by the almost superimposed red and green markers (which together appear yellow), whereas the left pinna rolled further medially.

The Up target sequence (Fig. 3) shows that the two pinnae moved asymmetrically, although the target was on the midline at $18^{\circ}$ above the horizontal. The position of the right pinna changed very little between the spontaneous and fixation periods, as seen by the overlap of the triangular markers. In the last part of the sequence, after the cat saccaded to the target, the lower position of the green reference dot indicates that the pinna pitched upward in the direction of the target. The small change in the position of the triangular markers, compared with the change in the position of the reference dot, indicates that the main rotation was accompanied by displacements that maintained the most distal part of the pinna in a relatively constant position as it pitched upward. The left pinna also showed little movement between the spontaneous and fixation stages but a more pronounced change in pitch in the upward direction of the distal end of the pinna, judging by the amount of downward displacement of the green markers.

These series of images illustrate (1) that the pinna moved in the general direction of the target; (2) that the movements of both pinnae were not necessarily symmetrical for horizontal or vertical targets; and (3) that in some instances, the pinna response was the result of combined complex rotations and displacements.

\section{Magnetic search coil recordings of pinna movements to visual targets}

Video recording of pinna movements (Figs. 2, 3) was done for illustrative purposes only; for routine recordings of pinna movements we used the magnetic search coil. Our coil system can only measure yaw (horizontal) and pitch (vertical) changes in coil position; thus it has limitations for the study of pinna movements because the structure moves in a complex manner in threedimensional space. The data in Figure 4, which were recorded during the videotaping session that produced the images shown in Figures 2 and 3, demonstrate that the two-dimensional search coil technique can provide a first approximation of the magnitude of pinna movements and precise information about their timing. The coil from which these recordings were obtained was implanted in Cat08's right pinna using the caudal approach (Fig. 1, left).

In all cases visual saccades started from a fixation LED at the primary position $\left(0^{\circ}, 0^{\circ}\right)$ to targets located at the four positions indicated above. The onset of the fixation LED usually occurred between -1200 and $-900 \mathrm{msec}$, depending on how long it took the cat to enter the fixation window. There was more variability in 


\section{Right Pinna (Cat08)}
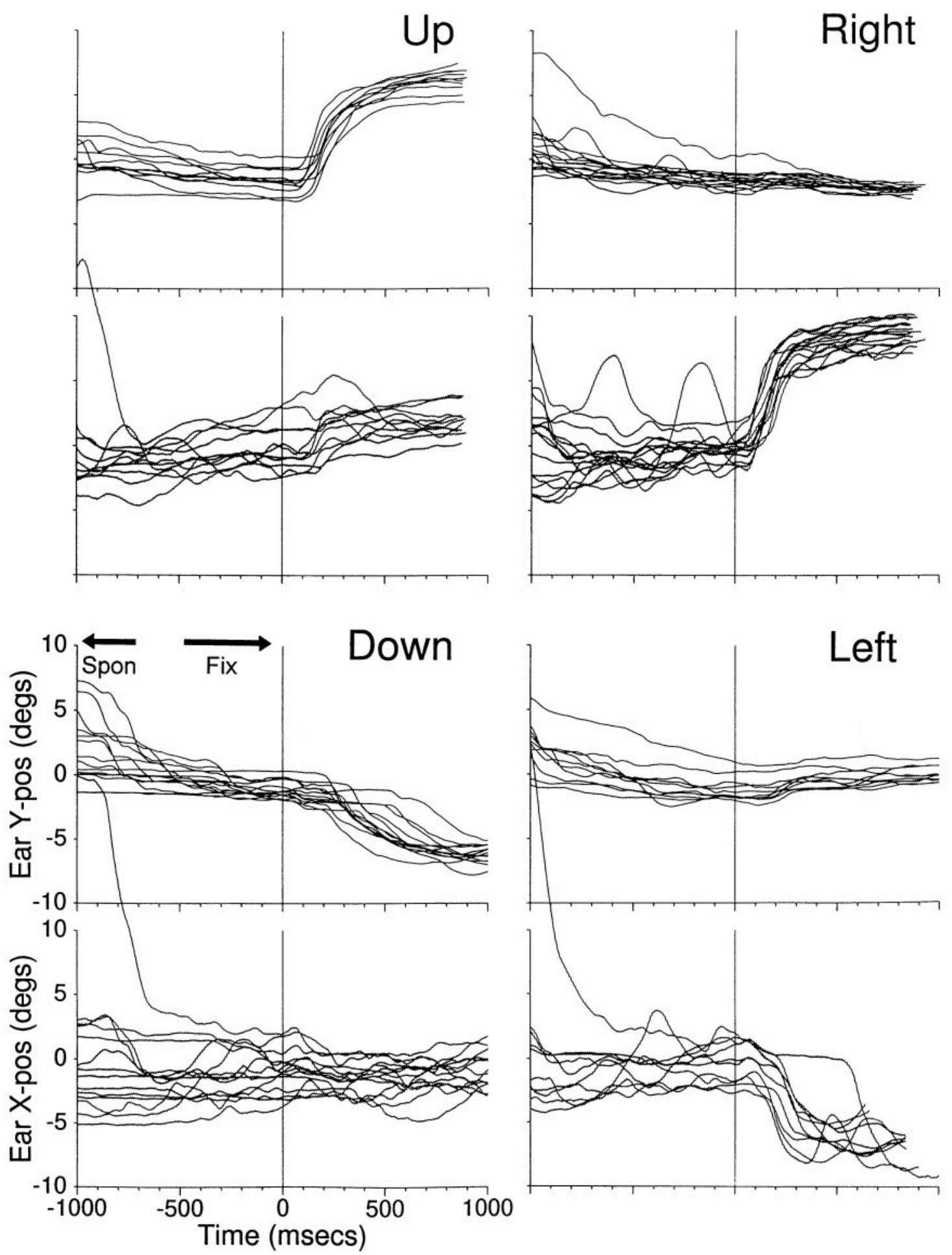

Figure 4. Pinna movement traces to visual targets from Cat08's right pinna. Each panel plots the vertical (top) and horizontal (bottom) component of pinna position synchronized to the onset of the target LED (lit for $1000 \mathrm{msec}$ ) at time $0 \mathrm{msec}$. The targets were located at $\left( \pm 18^{\circ}, 0^{\circ}\right),\left(0^{\circ}, 18^{\circ}\right)$, and $\left(0^{\circ},-23^{\circ}\right)$. Traces from several trials, including those shown in Figures 2 and 3, are plotted. The ordinates of these plots, as well as those of similar plots in subsequent figures, represent relative and not absolute pinna position. 


\section{Right Pinna (Cat09)}
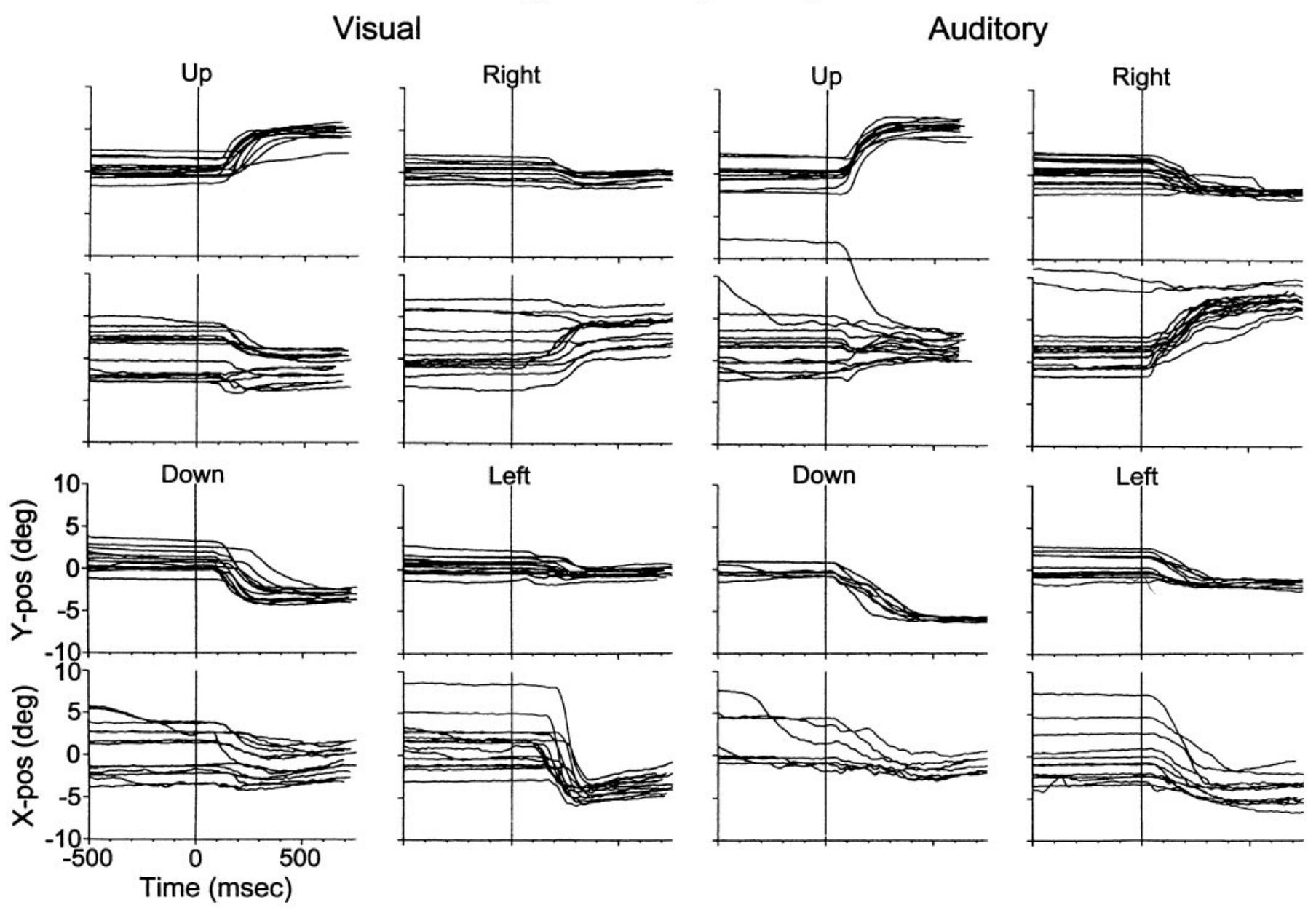

Figure 5. Movements of Cat09's right pinna evoked by visual (left) and auditory (right) targets recorded with the standard saccade task. The fixation LED was at the primary position $\left(0^{\circ}, 0^{\circ}\right)$ in all cases, and the targets were at $\left( \pm 18^{\circ}, 0^{\circ}\right),\left(0^{\circ}, 18^{\circ}\right)$, and $\left(0^{\circ},-14^{\circ}\right)$. All traces are plotted synchronized to the onset of the stimulus at Time, 0 msec.

pinna position during the spontaneous period before fixation onset than during the fixation period (between approximately -900 and $0 \mathrm{msec}$ ), indicating that the pinna adopted a more consistent position during the fixation of the LED at the primary position. This reduction in the spread is present in most traces. Notice that the pinnae did not assume random positions or seem to show periodic scanning movements.

The appearance of the visual targets evoked well defined consistent responses of the pinna in the direction of the sources. The amplitude of these movements did not match the eccentricity of the targets that evoked them: targets located $18^{\circ}$ or $23^{\circ}$ away from the primary position yielded pinna movements of $\sim 10^{\circ}$ (Fig. 4). Movements had both vertical and horizontal components, with the larger ones taking place along the axis on which the target was located. Finally, the movements were not symmetrical; movements of the right pinna were larger for the ipsilateral and upward targets than for the contralateral and downward targets, respectively. It is important to point out, however, that the movements measured by the coil depend on the placement of the coil on the pinna. For example, there are marked differences between these movements and those shown in Figure 5. Of course, these differences could also be attributable to differences in pinna movements between cats.

\section{Pinna movements to auditory and visual targets}

The pinna movement data shown in the previous figures were recorded during the course of standard saccade trials to visual stimuli presented within the cat's oculomotor range. In this section we compare pinna movements with both visual and auditory targets.

Plotted in Figure 5 are Cat09's right pinna movements recorded during standard saccade trials with a coil implanted in the caudal aspect of the structure (Fig. 1, left). The pinna movements that followed the onset of the visual stimulus were similar to those in Cat08 (Fig. 4); the largest component of movement was along the axis of the target and there was a similar asymmetry.

The broadband noise stimulus also evoked pinna movements in the direction of the sources (Fig. 5). Although these movements resemble those with equivalent visual targets, i.e., they are goaldirected and somewhat asymmetric, there are some marked differences between the pinna movements to visual and auditory targets. The major difference is that acoustically evoked movements have shorter latencies than those evoked by visual targets. The mean latency of the pinna movements to the visual target at $\left(18^{\circ}, 0^{\circ}\right)$ was $242.8 \mathrm{msec}(\mathrm{SD}, \pm 62.6 \mathrm{msec}$ ), whereas the mean to the auditory target at the same position was $34.6 \mathrm{msec}(\mathrm{SD}, \pm 15$ 


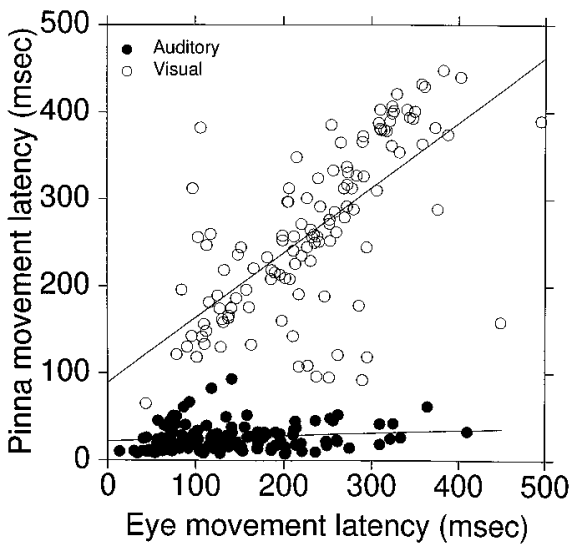

Figure 6. Pinna movement latency to auditory and visual targets. Measurements, taken from visual and auditory standard saccade trials to targets ipsilateral to the pinna at $\left( \pm 18^{\circ}, 0^{\circ}\right)$, are plotted as a function of eye movement latency. Data from five cats (Cat06, Cat09, Cat10, Cat11, and Cat14) are included; $n=249$ (126 auditory trials and 123 visual trials; 7 trials with latencies $>500 \mathrm{msec}$ were excluded).

msec). The mean latency of saccadic eye movements to the same visual target $(234.7 \mathrm{msec}$; SD, $\pm 33 \mathrm{msec}$ ) suggests that the eye and ear begin to move together and that pinna movements might be a part of a general orienting reflex.

We correlated the shortest pinna latency with eye movement latency in standard saccade trials to auditory and visual targets located on the ipsilateral side of the pinna at an eccentricity of $18^{\circ}$. Such data, from five different cats (Cat06, Cat09, Cat10, Cat11, and Cat14), are plotted in Figure 6. The mean latency of pinna movements to auditory targets is significantly shorter than the mean latency of pinna movements to visual targets $(p<$ $0.001)$. The slope of the regression line for auditory data $(0.03$; $r=0.16)$ indicates that there is no relationship between the onset of pinna (26.5 msec; SD, $15.2 \mathrm{msec}$ ) and eye (265.6 msec; SD, 77.8 msec) movements for these targets. On the other hand the slope of the line for the visual data $(0.75 ; r=0.68)$ indicates that the onset of eye (mean, $233.8 \mathrm{msec}$; SD, $87.2 \mathrm{msec}$ ) and pinna movements (262.8 msec; SD, $96.2 \mathrm{msec})$ are related.

\section{Pinna movements to delayed saccade and sensory probe tasks}

The results of Figure 6 suggest that pinna movements to auditory targets are coupled to the onset of the target, not to the eye movement. To confirm this observation, we also studied pinna movements using the delayed saccade task in which the eye movements are temporally dissociated from the target onset (Fig. 7). This allowed us to separate pinna movements that resulted from the presentation of a stimulus from those associated with the animal's orientation. The first part of the task, the fixation period before target presentation (time $<0$ ), was identical in both the visual and auditory conditions. After the eyes acquired the fixation $\mathrm{LED}$ at $\left(0^{\circ}, 0^{\circ}\right)$, the pinna remained in a stationary position that was generally consistent from trial to trial. During the delay period the behavior of the eyes was similar in both visual and auditory conditions; they remained on the fixation LED, but the pinna behaved differently. In visual trials the pinna remained stationary during the entire $500 \mathrm{msec}$ period in which the fixation light and the target overlapped and moved at about the same time the eyes started to move to the target after the fixation LED was turned off. In auditory trials the response of the pinna exhibited two components: one prominent and abrupt with very short latency (Fig. 7, filled arrows; mean, $21 \mathrm{msec}$; SE, $\pm 2.2 \mathrm{msec}$ ) that was time-locked to target onset and another smaller and later at approximately the time of eye movement onset (open arrows).

The short-latency component was also revealed by the auditory sensory probe task, which required the cat to maintain fixation on an LED without an eye movement, thereby excluding the second component of the pinna response. To the cat the initial segments of delayed saccade (Fig. 7) and sensory probe (Fig. 8) trials are identical. Because eye movements to the target were not required, these sensory probe trials also enabled us to measure pinna movements to more eccentric targets, outside the cat's oculomotor range.

Figure 8 illustrates Cat14's pinna movements of both the left and right ears recorded with coils implanted with the caudal approach. The noise stimuli evoked prominent and consistent short-latency pinna movements that were largest for stimuli on the ipsilateral side. Note that the movements of the pinnae were graded as a function of speaker position, with the largest and smallest movements evoked by the most eccentric ipsilateral and contralateral probes, respectively. In these trials, the duration of the noise stimulus was $800 \mathrm{msec}$, and the reward was delivered $200 \mathrm{msec}$ after the noise was turned off. Note that the large pinna movements to the most eccentric stimuli returned toward the central position after the offset of the noise.

We measured the latencies of pinna movements evoked by acoustic stimuli presented from different positions along the horizontal plane when the cat was fixating the LED at $\left(0^{\circ}, 0^{\circ}\right)$ and did not move its eyes (Fig. 8). Figure 9 includes results from Cat14's data shown in Figure 8 and from Cat11. Cat14's mean response latencies across all speaker positions were $24.5 \mathrm{msec}(\mathrm{SD}, \pm 9.7$ msec; $n=176$ ) for the left pinna and $23.4 \mathrm{msec}(\mathrm{SD}, \pm 11.1 \mathrm{msec}$; $n=166$ ) for the right ear. The latencies were significantly longer for the most peripheral targets compared with the central one on both the ipsilateral and contralateral side, as evidenced by the lack of overlap of the confidence intervals in Figure 9.

Another characteristic of the short-latency component was its lack of habituation. In Cat06, the subject with the longest tenure in our studies, it was observed after $>10^{5}$ trials.

\section{Pinna movements are goal-oriented}

All cats that participated in our studies showed pinna movements in both visual and auditory trials that appeared to be goaloriented. The data presented in Figures 2-6 show that the cats moved their pinnae purposely and consistently toward the stimulus. Furthermore, they seemed to be moving the ear to a particular position, regardless of the initial pinna position, as illustrated by the adjustments the cat made in some of the trials shown in Figure 5, in which the pinna was clearly in an anomalous position during the fixation period. In these trials the pinna was not in its usual position at the time the visual or auditory target came on, and the resulting pinna movement compensated for the unusual initial position, in some cases by moving in the opposite direction from the majority of the movements. Similar observations are found among the trials shown in Figure 8.

The consistent position of the pinna during the fixation period in the data shown in Figure 7 allowed us to compare the amplitude of the movements between the auditory and visual conditions. The mean amplitude of the pinna movements shown in Figure 7 to targets at $\left(18^{\circ}, 0^{\circ}\right)$ and $\left(9^{\circ}, 0^{\circ}\right)$ are plotted in Figure 10 . The overlap of the confidence intervals suggests that the final pinna position in visual and auditory trials is similar, given a 


\section{Right Pinna (Cat06)}
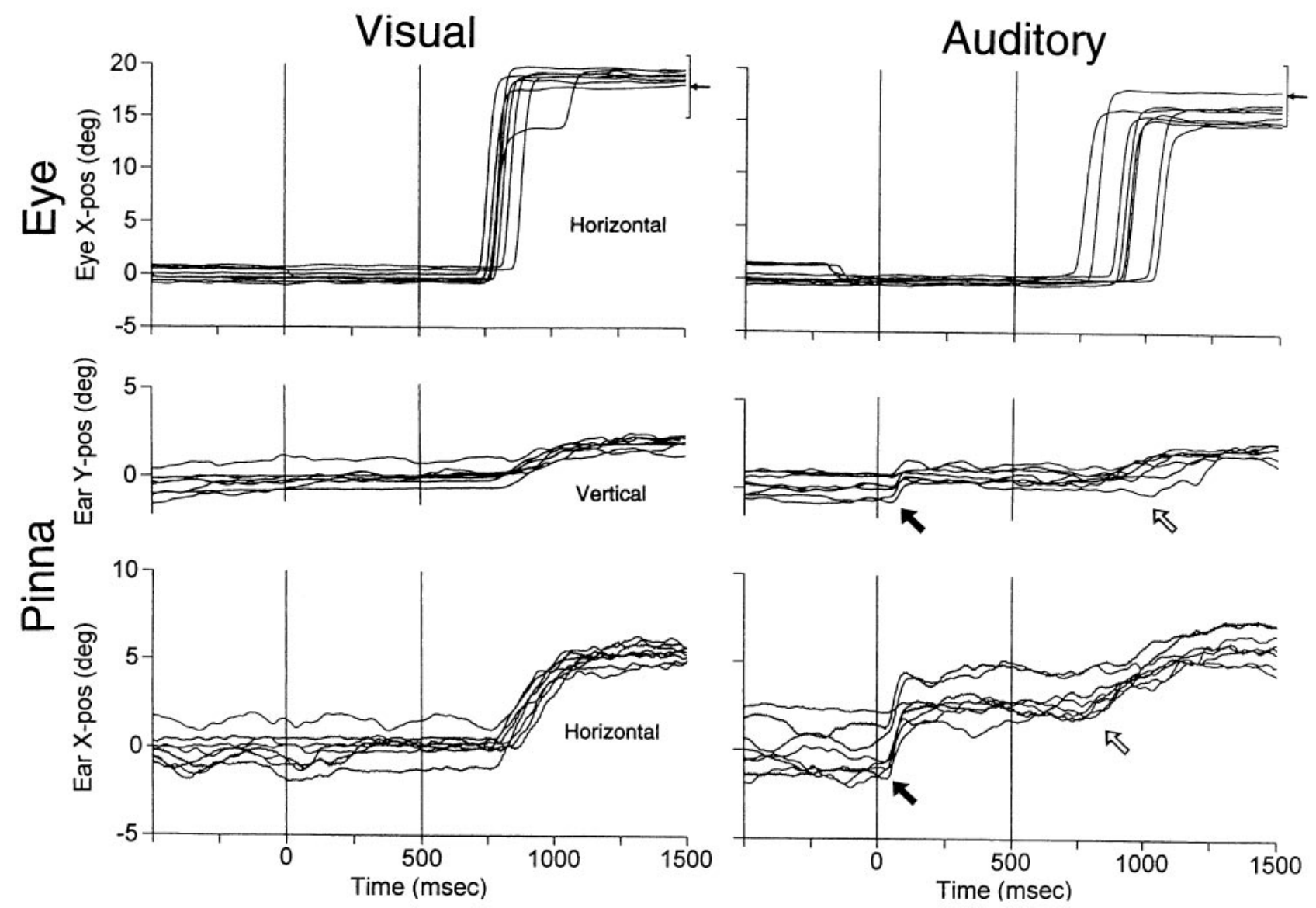

Figure 7. Pinna movements in delayed saccade trials. Visual and auditory targets were located at $\left(18^{\circ}, 0^{\circ}\right)$. The coil was implanted on the flat aspect of Cat06's right pinna (Fig. 1, right). From top to bottom: Horizontal eye position and Vertical and Horizontal pinna position. Delay duration, 500 msec. Notice the short-latency (solid arrows) and long-latency (open arrows) pinna movements in the auditory condition; $n=16$ ( 8 visual and 8 auditory trials).

consistent starting position. Notice that in both cases the change in position is a weak function of target eccentricity.

\section{Pinna movements to visual targets are not the result of training}

Because our experimental design incorporates both auditory and visual trials (Populin and Yin, 1998), we were able to study the behavior of the pinna while the cat oriented to nonacoustic targets. Our findings confirm the observations of Joseph and Boussaud (1985), who reported that cat eye movements to visual targets were accompanied by electromyographic discharges in pinna muscles.

The consistency of the pinna movements to visual targets raised the possibility that they might be the result of some aspect of the training program used to teach cats to look at the location of sound sources (Populin and Yin, 1998). We examined the first trial of the first experimental session of three subjects (Cat09, Cat11, and Cat15), and in all cases the cats moved their pinnae in the direction of the visual target as they oriented.

\section{DISCUSSION}

\section{Methodological considerations}

Both the limitations and the strengths of this study concern the use of the magnetic search coil. The technique is restrictive for measuring pinna movements because (1) the structure moves in three dimensions, whereas our coil system measures movements around two axes only; and (2) the position of the coil on the pinna places it outside of the linear range of the system. On the other hand, with the system interfaced to a computer that allows fast sampling rates, pinna movements can be recorded with a time resolution not available with conventional video.

\section{Coil calibration}

As described in Materials and Methods, the magnetic search coil system is optimal when the coil is positioned in the coronal plane. Anatomical considerations, however, prevent the pinna coils from being implanted in that position. Therefore, we took advantage of the cat's habit of orienting the pinnae in a standard position while fixating an LED at the primary position and made our calibrations about that position. Such a procedure is essential for proper calibration. Previous measurements of cat pinna movements using the search coil technique by Hartline et al. (1995) have not mentioned any calibration procedure so it is difficult to determine how they converted the voltage output of the coil system to degrees.

\section{Pinna movements and two-dimensional movement recordings}

The limitations of the calibration procedure, coupled with the uncertainty along which axis the pinna is moving, limit our interpretation of the measurements to relative changes in pinna 

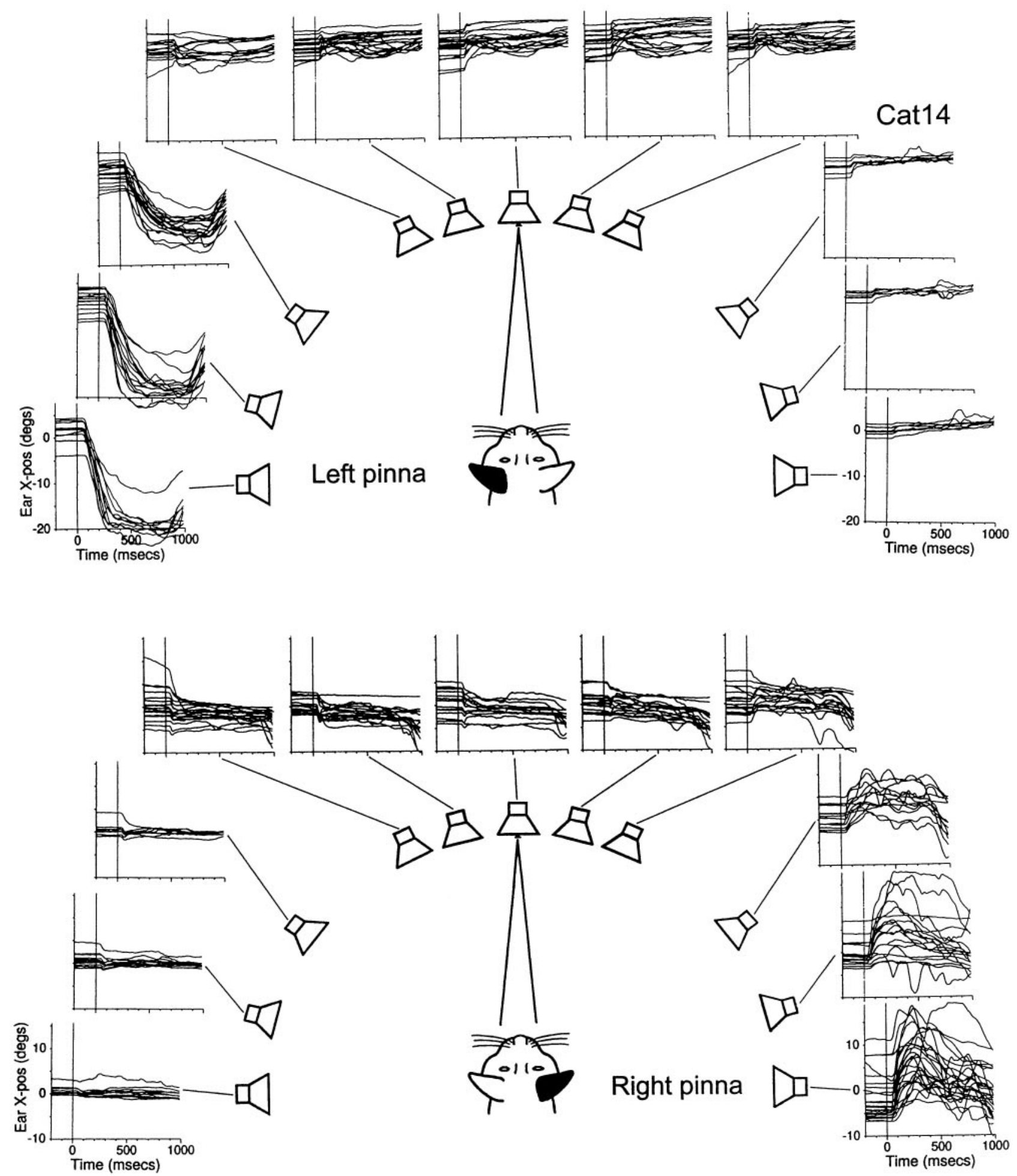

Figure 8. Short-latency component of pinna movements during sensory probe trials. Acoustic broadband (800 msec, broadband noise) probes were presented in random trials from speakers located at $\left( \pm 90^{\circ}, 0^{\circ}\right),\left( \pm 63^{\circ}, 0^{\circ}\right),\left( \pm 45^{\circ}, 0^{\circ}\right),\left( \pm 18^{\circ}, 0^{\circ}\right),\left( \pm 9^{\circ}, 0^{\circ}\right)$, and $\left(0^{\circ}, 0^{\circ}\right)$ while the cat fixated an $\mathrm{LED}$ at $\left(0^{\circ}, 0^{\circ}\right)$. The end of the movement traces coincided with the delivery of the reward 200 msec after stimulus offset. Notice in the conditions with the largest movements that the pinna returns to the initial position shortly after stimulus offset. For both left and right pinnae the trials corresponding to contralateral speaker positions at $\pm 90^{\circ}, \pm 63^{\circ}$, and $\pm 45^{\circ}$ were recorded in a different session than the rest of the data.

position along the yaw and pitch axes. The simultaneous recordings of pinna movements with the magnetic search coil and videotape indicate that the main aspects of the movements are nevertheless recorded.
On the other hand, we are confident of our measurements of the timing of pinna movements. In this case, the actual position of the coil, not quite in the coronal plane, worked to our advantage, because rolling (lateral) movements of the pinna also produce 
Figure 9. Latencies of pinna movements evoked by broadband long-duration acoustic stimuli during sensory probe trials. The bars represent confidence intervals $(2 \times$ SEM). Cat14, 342 trials (132 left pinna, 210 right pinna), raw data are shown in Figure 8. Cat11, 195 trials (93 left pinna, 102 right pinna).

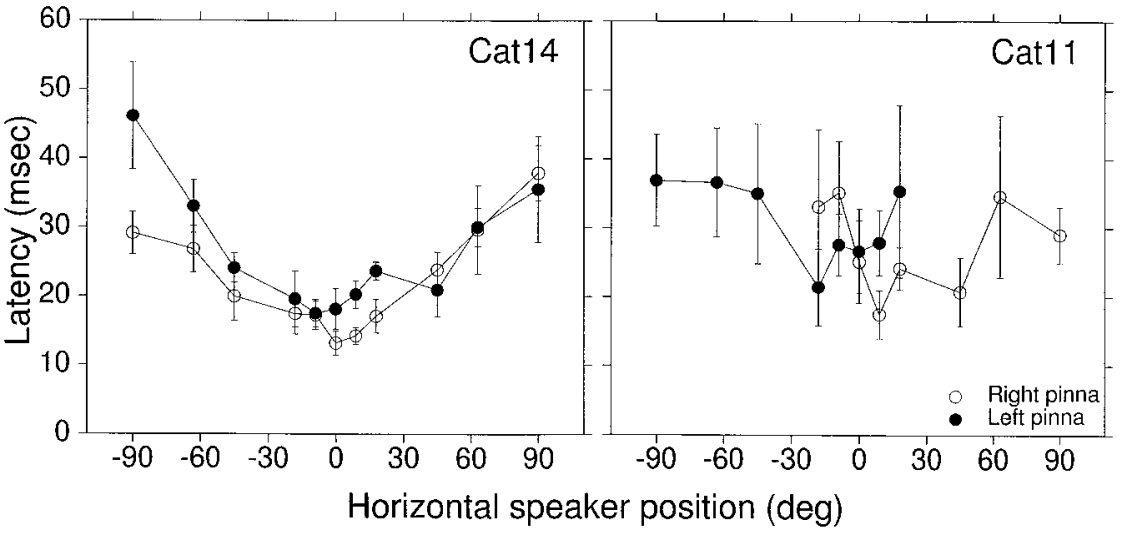

prepared to localize sound, pulled their pinnae to a position that provided some acoustical advantage. Bringing the pinnae to a standard position when certain of having to localize the source of a sound could be a simplifying strategy that could facilitate localization (Young et al., 1996).

The question, therefore, arises as to whether a standard pinna position is required for accurate localization. Our sound localization data show that cats are able to localize acoustic targets starting from different fixation positions (Populin, 1996; Populin and Yin, 1997b) that are associated with different pinna positions, thus indicating that a standard pinna position is not required for accurate localization. Furthermore, the amplitude of pinna movements in our data range up to $\sim 20^{\circ}$, which is within the range of different ear positions studied by Young et al. (1996). Therefore, we would expect that our cats experienced similar changes in acoustic input from stationary sources as illustrated in their study.

Figure 10. Relative changes in pinna position evoked by visual (hollow symbols) and auditory (filled symbols) targets located at $\left(18^{\circ}, 0^{\circ}\right)$ and $\left(9^{\circ}, 0^{\circ}\right)$ in delayed saccade trials; data from Figure 7 are included. The mean pinna position at the start of the trials (filled star) has been arbitrarily set at coordinates $\left(0^{\circ}, 0^{\circ}\right)$. Circles represent the change in pinna position for the targets at $\left(9^{\circ}, 0^{\circ}\right)$; triangles represent the change in pinna position for the targets at $\left(18^{\circ}, 0^{\circ}\right)$; and the standard bars represent $\pm 2 \mathrm{SE}$ of the sample mean; $n=62$ (23 visual and 39 auditory trials).

movement components of yaw and pitch, which we can detect. Thus it is unlikely that we missed any pinna movements, although we cannot claim to determine their precise type.

\section{Pinna movements during sound localization}

The primary goal of this study was to characterize the behavior of the cat's pinna during sound localization. When the cat anticipated that the start of a trial was imminent, it looked toward the center and moved its pinnae to a ready position. A similar behavior has been reported by May and Huang (1996) in the conditioned head-free cat before the presentation of a target and by Heffner and Heffner (1982) in an elephant trained in a left-right discrimination task. Just before starting a trial, the elephant extended its pinnae perpendicularly to its head, to return them to their resting position while executing the response with its trunk. Significantly, this pinna behavior was not observed during threshold experiments conducted before and after the discrimination experiment, suggesting that it was specific to the task that required a localization judgment.

The extent to which the behavior of the cats of May and Huang (1996) and the elephant of Heffner and Heffner (1982) is analogous to the behavior of our cat's pinnae is debatable given the differences between the species and experimental paradigms, but the similarities are intriguing. It appears as if both species, as they

\section{Neural mechanisms of pinna control}

Pinna movements to auditory targets are stereotyped and consistent, goal-oriented, and have shorter latencies than to corresponding visual targets. They consist of two parts: a short-latency component time-locked to the onset of the sound and a second long-latency component that is highly correlated with the eye movement and may be part of the animal's general orientation behavior (Schaefer, 1970; Stein and Clamann, 1981; Hartline et al., 1995).

The distinct characteristics of each component of pinna movement suggest that they may be controlled separately. The close association between eye and pinna movements to visual targets is consistent with electrical stimulation of the SC, which evokes coordinated movements of the eyes, pinna, and whiskers (Stein and Clamann, 1981). These movements could be mediated by the tectoreticular-facial pathway or the tectoparalemniscal-facial pathways (May et al., 1990). The paralemniscal area, in the lateral midbrain tegmentum of the cat (Henkel and Edwards, 1978; Henkel, 1981), supplies a large and elaborate network of monosynaptic excitatory and inhibitory inputs to the medial aspect of the facial nucleus (May et al., 1990), where the motoneurons that innervate the muscles of the pinna are located (Kume et al., 1978; Populin and Yin, 1995).

The role of the SC in the control of the short-latency component described above is not as clear, however. The average latency with which the right pinna begins to move in response to acoustic stimuli presented on the frontal hemifield (Fig. 7) is 23.5 msec. This time seems too short to include the intermediate and deep SC, the units of which respond to acoustic stimuli with an average first spike latency of $19 \mathrm{msec}$ in the same preparation 
performing the same sensory probe task (Populin and Yin, 1997b). Longer auditory SC latencies have also been reported in the behaving cat (Peck et al., 1995) and monkey (Jay and Sparks, 1987) (median, $50 \mathrm{msec}$; mean, $44.8 \mathrm{msec}$, respectively). Latency measurements of electrically evoked pinna movements, which could shed some light onto this issue, are not reported in the literature.

If the SC were not involved in the generation of the shortlatency component of pinna movements, then what pathways could underlie this behavior? The goal-oriented nature of the short-latency component suggests that the paralemniscal zone may be involved. Auditory inputs to this area seem to be limited to the nucleus sagulum (Henkel, 1981), which is considered part of the auditory system but has not been studied physiologically. Alternatively, it is possible that auditory inputs reaching the medial aspects of the facial nucleus, bypassing the paralemniscal zone, could drive these movements. Pinna movement-related activity has been demonstrated in units in the pontomedullary reticular formation of the cat (Siegel et al., 1980). Stapedius motoneurons are located near the facial nucleus and receive direct inputs from the medial superior olive (Borg, 1973; Joseph et al., 1985). Thus auditory information does reach the vicinity of the facial nucleus, but the existence of the required synaptic contacts to complete the short-latency pinna movement circuit remains to be demonstrated.

\section{Summary and conclusions}

The results of this study show that cat pinna movements during sound localization consist of a short-latency component, timelocked to the onset of the stimulus, and a second component that accompanies the eye movement to the target. The consistency of the behaviors observed and the changes in acoustics that can result from them (Phillips et al., 1982; Musicant et al., 1990; Rice et al., 1992; Young et al., 1996) raise the question of what the cat accomplishes by moving its pinna. The two different types of pinna movements observed suggest that various roles could be fulfilled. With the short-latency component the cat could (1) obtain multiple samples of an acoustic object within a short period, which could help in localization (Thurlow and Runge, 1967), and (2) separate the spectrum of a sound source from the HRTF (Young et al., 1996). On the other hand, the larger pinna movements observed during an orientation response could help improve signal-to-noise ratio by focusing the acoustic axis of the pinna on a particular area of space (Phillips et al., 1982).

\section{REFERENCES}

Borg E (1973) On the neuronal organization of the acoustic middle ear reflex. A physiological and anatomical study. Brain Res 49:101-123.

Calford MB, Pettigrew JD (1984) Frequency dependence of directional amplification at the cat's pinna. Hear Res 14:13-19.

Fuchs AF, Robinson DA (1966) A method for measuring horizontal and vertical eye movement chronically in the monkey. J Appl Physiol 21:1068-1070.

Hartline PH, Pandey Vimal RL, King AJ, Kurylo DD, Northmore DPM (1995) Effects of eye position on auditory localization and neural representation of space in superior colliculus of cats. Exp Brain Res 104:402-408.

Heffner RS, Heffner HE (1982) Hearing in the elephant (Elephas maxi$m u s$ ): absolute sensitivity, frequency discrimination, and sound localization. J Comp Physiol Psychol 96:926-944.

Heffner RS, Heffner HE (1988) Sound localization acuity in the cat: effect of azimuth, signal duration, and test procedure. Hear Res $36: 221-232$.
Henkel CK (1981) Afferent sources of a lateral midbrain tegmental zone associated with the pinnae in the cat as mapped by retrograde transport of horseradish peroxidase. J Comp Neurol 203:213-226.

Henkel CK, Edwards SB (1978) The superior colliculus control of pinna movements in the cat: possible anatomical connections. J Comp Neurol 182:763-776.

Jay MF, Sparks DL (1987) Sensorimotor integration in the superior colliculus. II. Coordinates of auditory signals. J Neurophysiol 57:35-55.

Jenkins WM, Masterton RB (1982) Sound localization: effects of unilateral lesions in central auditory system. J Neurophysiol 47:987-1016.

Joseph JP, Boussaud D (1985) Role of the cat substantia nigra pars reticulata in eye and head movements I. Neural activity. Exp Brain Res 57:286-296.

Joseph MP, Guinan JJ, Fullerton BC, Norris BE, Kiang NYS (1985) Number and distribution of stapedius motoneurons in cats. J Comp Neurol 232:43-54.

Kume M, Uemura M, Matsuda K, Matsushima R, Mizuno N (1978) Topographical representation of peripheral branches of the facial nerve within the facial nucleus: a HRP study in the cat. Neurosci Lett 8:5-8.

May BJ, Huang AY (1996) Sound orientation behavior in cats. I. Localization of broadband noise. J Acoust Soc Am 100:1059-1069.

May PJ, Vidal P-P, Baker R (1990) Synaptic organization of tectal-facial pathways in cat II. Synaptic potentials following midbrain tegmentum stimulation. J Neurophysiol 64:381-402.

Middlebrooks JC (1992) Narrow-band sound localization related to external ear acoustics. J Acoust Soc Am 92:2607-2624.

Middlebrooks JC, Knudsen EI (1987) Changes in external ear position modify the spatial tuning of auditory units in the cat's superior colliculus. J Neurophysiol 57:672-687.

Musicant AD, Chan JCK, Hind JE (1990) Direction-dependent spectral properties of cat external ear: new data and cross-species comparisons. J Acoust Soc Am 87:757-781.

Peck CK, Baro JA, Warder SM (1995) Effects of eye position on saccadic eye movements and on the neuronal responses to auditory and visual stimuli in cat superior colliculus. Exp Brain Res 103:227-242.

Phillips DP, Brugge JF (1985) Progress in neurophysiology of sound localization. Annu Rev Psychol 36:245-274.

Phillips DP, Calford MB, Pettigrew JD, Aitkin LM, Semple MN (1982) Directionality of sound pressure transformation at the cat's pinna. Hear Res 8:13-28.

Populin LC (1996) Sound localization and sensorimotor integration in the behaving cat. PhD thesis, University of Wisconsin-Madison.

Populin LC, Yin TCT (1995) Topographical organization of the motoneuron pools that innervate the muscles of the pinna of the cat. J Comp Neurol 363:600-614.

Populin LC, Yin TCT (1997a) Pinna movements during sound localization in the behaving cat. Assoc Res Otolaryngol 205:52.

Populin LC, Yin TCT (1997b) Sensitivity of auditory cells in the superior colliculus to eye position in the behaving cat. In: Psychophysical and physiological advances in hearing (Palmer AR, Rees A, Summerfield AQ, Meddis R, eds), pp 441-448. London: Whurr.

Populin LC, Yin TCT (1998) Behavioral studies of sound localization in the cat. J Neurosci 18:2147-2160.

Rice JJ, May BJ, Spirou GA, Young ED (1992) Pinna-based spectral cues for sound localization in cat. Hear Res 58:132-152.

Schaefer KP (1970) Unit analysis and electrical stimulation in the optic tectum of rabbits and cats. Brain Behav Evol 3:222-240.

Siegel JM, Wheeler RL, Breedlove SM, Mcginty DJ (1980) Brain stem units related to movements of the pinna. Brain Res 202:183-188.

Stein BE, Clamann HP (1981) Control of pinna movements and sensorimotor register in cat superior colliculus. Brain Behav Evol 19:180-192.

Thurlow WR, Runge PS (1967) Effect of induced head movements on localization of direction of sounds. J Acoust Soc Am 42:480-488.

Wiener FM, Pfeiffer RR, Backus SN (1966) On the sound pressure transformation by the head and auditory meatus of the cat. Acta Otolaryngol 61:255-269.

Wightman FL, Kistler DJ (1989) Headphone simulation of free-field listening. I: Stimulus synthesis. J Acoust Soc Am 85:858-867.

Young ED, Rice JJ, Tong SC (1996) Effects of pinna position on headrelated transfer functions in the cat J Acoust Soc Am 99:1-13. 